\title{
Stochastic Optimal Control of Structural Systems
}

\author{
Z.G. Ying*
}

Department of Mechanics, Zhejiang University, Hangzhou 310027, P. R. China

\begin{abstract}
The stochastic optimal control is an important research subject in structural engineering. Recently, a stochastic optimal nonlinear control method has been proposed based on the stochastic dynamical programming principle and stochastic averaging method. The active and semi-active stochastic optimal control methods have been further developed for structural systems. The control saturation or bound, partial state observation, etc. have been taken into account by the stochastic optimal control. The present paper surveys these research developments.
\end{abstract}

\section{INTRODUCTION}

The strong nonlinear stochastic vibration of engineering structures such as tall buildings and large bridges is frequently caused by severe loadings such as earthquake and storm. The stochastic optimal control is an important research subject in structural engineering [1]. The mathematics theory of stochastic optimal control has been developed [24]. However, the linear-quadratic-Gauss (LQG) control method has mostly used for structural vibration control [5]. Recently, some stochastic optimal nonlinear control methods $[6,7]$ have been proposed, in particular, the method based on the stochastic dynamical programming principle and stochastic averaging method [8-17]. This control method can achieve better control effectiveness and efficiency than the LQG control method. These research developments are surveyed as follows: (1) stochastic optimal control law; (2) optimal active control; (3) optimal semi-active control; (4) developments of stochastic optimal control method.

\section{STOCHASTIC OPTIMAL CONTROL LAW [8, 9]}

A controlled and stochastically excited nonlinear structural system with multi-degree-of-freedom can be expressed as

$$
\boldsymbol{M} \ddot{\boldsymbol{X}}+\boldsymbol{C}(\boldsymbol{X}, \dot{\boldsymbol{X}}) \dot{\boldsymbol{X}}+\frac{\partial V_{s}(\boldsymbol{X})}{\partial \boldsymbol{X}}=\boldsymbol{F}(\boldsymbol{X}, \dot{\boldsymbol{X}}) \boldsymbol{W}(t)+\boldsymbol{B} \boldsymbol{U}
$$

where $\boldsymbol{X}$ is the $n$-dimensional structural displacement vector, $V_{s}(\boldsymbol{X})$ is the structural potential, $\boldsymbol{W}(t)$ is the stochastic excitation vector, $\boldsymbol{U}$ is the control force vector. Rewrite Eq. (1) in the following form of quasi Hamiltonian equation:

$$
\begin{aligned}
\dot{Q}_{i} & =\frac{\partial H}{\partial P_{i}} \\
\dot{P}_{i} & =-\frac{\partial H}{\partial Q_{i}}-c_{i j} \frac{\partial H}{\partial P_{j}}+f_{i k} W_{k}(t)+u_{i}
\end{aligned}
$$

*Address correspondence to this author at the Department of Mechanics, Zhejiang University, Hangzhou 310027, P.R. China;

E-mail: yingzg@zju.edu.cn where $H$ is the Hamiltonian function, $Q_{i}$ and $P_{i}$ are respectively displacement and momentum, $W_{k}(t)$ is the stochastic excitation, assumed as Gaussian white noise, $u_{i}$ is the control.

Apply the stochastic averaging method to system (2) yields averaged Itô stochastic differential equations. In the integrable and non-resonant case, for instance, the Itô equation is

$$
d H_{r}=\left[m_{r}(\boldsymbol{H})+<u_{i} \frac{\partial H_{r}}{\partial P_{i}}>\right] \mathrm{d} t+\sigma_{r s}(\boldsymbol{H}) \mathrm{d} B_{s}(t)
$$

where $H_{r}$ is the first integral of Hamiltonian system, $m_{r}$ and $\sigma_{r s}$ are respectively the averaged drift and diffusion coefficients, $B_{s}(t)$ is the unit Wiener process, $\langle\cdot\rangle$ is the average operator. The objective of stochastic optimal control is to design a control $u_{i}$ which minimizes the performance index of system (2) or (3). For finite time-interval response control, the performance index is

$$
J=E\left[\int_{0}^{t_{f}} L\left(H_{r}, u_{i}\right) \mathrm{d} t+\Psi\left(H_{r}\left(t_{f}\right)\right)\right]
$$

and for infinite time-interval ergodic control, it is

$$
J=\lim _{t_{f} \rightarrow \infty} \frac{1}{t_{f}} \int_{0}^{t_{f}} L\left(H_{r}, u_{i}\right) \mathrm{d} t
$$

where $L$ is the performance function, $\Psi$ is the terminal cost. Eqs. (3) and (4) constitute a stochastic optimal control problem. By using the stochastic averaging method, the dimension of system equations can been reduced, the dynamical programming equation becomes non-degenerate and the response control is converted into the energy control.

According to the stochastic dynamical programming principle, the dynamical programming equation can been built, for system (3) and index (4b) as

$$
\min _{u_{i}}\left\{L\left(H_{r}, u_{i}\right)+\left[m_{r}+<u_{i} \frac{\partial H_{r}}{\partial P_{i}}>\right] \frac{\partial V}{\partial H_{r}}+\frac{1}{2} \sigma_{r k} \sigma_{s k} \frac{\partial^{2} V}{\partial H_{r} \partial H_{s}}\right\}=\lambda
$$


where $V$ is the value function. Then the optimal control law can be obtained by minimizing the left side of Eq. (5). Let function $L$ be

$$
L\left(H_{r}, u_{i}\right)=g\left(H_{r}\right)+<R_{i j} u_{i} u_{j}>
$$

The optimal control is

$$
u_{i}^{*}=-\frac{1}{2} R_{i j}^{-1} \frac{\partial H_{r}}{\partial P_{j}} \frac{\partial V}{\partial H_{r}}
$$

where $\partial H_{r} / \partial P_{j}$ is a linear function of velocity generally so that $u_{i}{ }^{*}$ is a quasi-linear damping force. The value function $V$ is obtained by solving the following equation:

$$
\begin{aligned}
& \frac{1}{2} \sigma_{r k} \sigma_{s k} \frac{\partial^{2} V}{\partial H_{r} \partial H_{s}}+m_{r} \frac{\partial V}{\partial H_{r}}-\frac{1}{4} R_{i j}^{-1}<\frac{\partial H_{r}}{\partial P_{i}} \frac{\partial H_{s}}{\partial P_{j}} \frac{\partial V}{\partial H_{r}} \frac{\partial V}{\partial H_{s}}> \\
& +g\left(H_{r}\right)-\lambda=0
\end{aligned}
$$

The stochastic optimal control method above has been applied to a single-degree-of-freedom system with cubic nonlinearity and two-degree-of-freedom nonlinear system under Gaussian white noise and seismic excitations. Numerical results show that the control effectiveness and efficiency by using the proposed method are better than by using the LQG method in terms of root-mean-square response (Figs. 1 and 2).

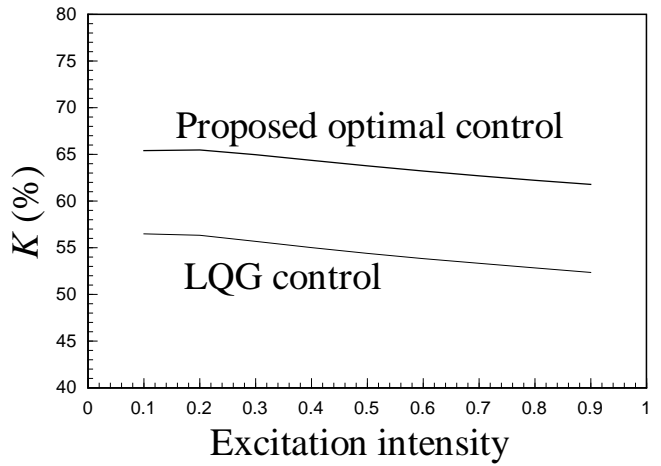

Fig. (1). Control effectiveness ( $K$-percentage reduction in rootmean-square displacements).

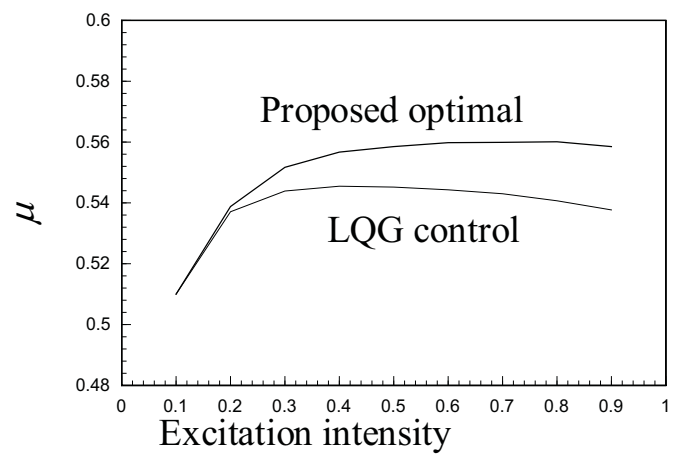

Fig. (2). Control efficiency ( $\mu$-ratio of control effectiveness to normalized root-mean-square control).

\section{OPTIMAL ACTIVE CONTROL $[10,11]$}

The optimal feedback control (7) can be implemented by active control devices, for instance, active mass damper (AMD). The optimal active control method has been applied to the vibration control of a hysteretic column system and coupled adjacent structures.

\section{Optimal Active Control of Hysteretic Column}

A controlled and parametrically/externally excited hysteretic column system can be expressed as

$$
\begin{aligned}
& \ddot{X}+2 \zeta \dot{X}+\left[\alpha-k_{1}-k_{2} \eta(t)\right] X+(1-\alpha) Z=\xi(t)+u \\
& \dot{Z}=A \dot{X}-\beta \dot{X}|Z|^{n}-\gamma|\dot{X}| Z|Z|^{n-1}
\end{aligned}
$$

where $X$ is the dimensionless horizontal displacement, $Z$ is the hysteretic force satisfying BW hysteresis Eq. (9b), $\eta(t)$ and $\xi(t)$ are respectively the vertical parametric excitation and horizontal external excitation, assumed as Gaussian white noises, $u$ is the control. Firstly, the hysteretic force $Z$ is separated equivalently into quasi-linear damping force and nonlinear elastic restoring force to yield equivalent nonhysteretic system

$$
\ddot{X}+\left[2 \zeta+2 \zeta_{1}(H)\right] \dot{X}+\frac{\partial U_{1}}{\partial X}=\xi(t)+k_{2} X \eta(t)+u
$$

where $H$ is the total system energy. Then applying the stochastic averaging method yields averaged Itô stochastic differential equation

$$
d H=\left[m(H)+<u \frac{\partial H}{\partial \dot{X}}>\right] \mathrm{d} t+\sigma(H) \mathrm{d} B(t)
$$

According to the stochastic dynamical programming principle, the dynamical programming equation for system (11) and index (4b) is obtained as

$$
\min _{u}\left\{L(H, u)+\left(m+<u \frac{\partial H}{\partial P}>\right) \frac{\partial V}{\partial H}+\frac{1}{2} \sigma^{2} \frac{\partial^{2} V}{\partial H^{2}}\right\}=\lambda
$$

For the function $L$ quadratic in control, the optimal active control is

$$
u^{*}=-\frac{1}{2 R} \frac{\partial V}{\partial H} \frac{\partial H}{\partial \dot{X}}=-\frac{1}{2 R} \frac{\partial V}{\partial H} \dot{X}
$$

The response statistics of the controlled system can be evaluated by substituting the optimal control into Eq. (9a) and using the stochastic averaging method. Numerical results are shown in Fig. (3).

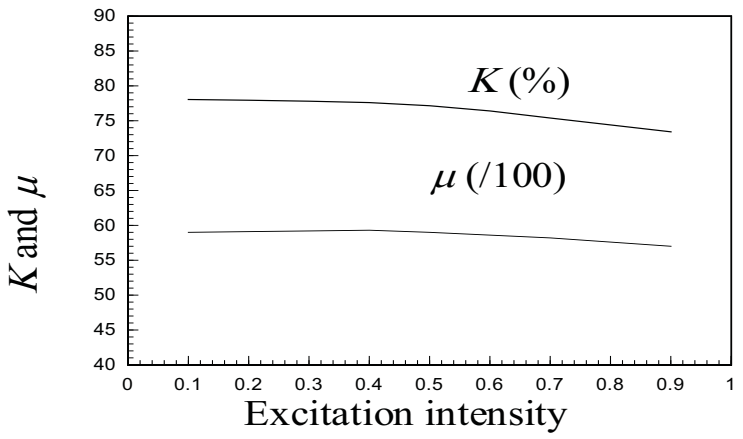

Fig. (3). Control effectiveness ( $K$-percentage reduction in rootmean-square displacements) and efficiency ( $\mu$-ratio of control effectiveness to normalized root-mean-square control) of the hysteretic column. 


\section{Optimal Active Control of Coupled Adjacent Structures}

A controlled and coupled multi-storey structures system under seismic ground motion excitation can be expressed as

$$
\begin{aligned}
& \boldsymbol{M}_{1} \ddot{\boldsymbol{X}}_{1}+\boldsymbol{C}_{1} \dot{\boldsymbol{X}}_{1}+\boldsymbol{K}_{1} \boldsymbol{X}_{1}=-\ddot{x}_{g}(t) \boldsymbol{M}_{1} \boldsymbol{E}_{1}+\boldsymbol{P}_{1} \boldsymbol{U} \\
& \boldsymbol{M}_{2} \ddot{\boldsymbol{X}}_{2}+\boldsymbol{C}_{2} \dot{\boldsymbol{X}}_{2}+\boldsymbol{K}_{2} \boldsymbol{X}_{2}=-\ddot{x}_{g}(t) \boldsymbol{M}_{2} \boldsymbol{E}_{2}+\boldsymbol{P}_{2} \boldsymbol{U}
\end{aligned}
$$

where $\boldsymbol{X}_{1}$ and $\boldsymbol{X}_{2}$ are respectively the displacement vectors of structures 1 and 2, $\ddot{x}_{g}(t)$ is the seismic excitation with the KT power spectral density, $\boldsymbol{U}$ is the coupling control force vector. By the structural mode transforming, reducing and stochastic averaging, the Itô stochastic differential equation obtained is

$$
\mathrm{d} \overline{\boldsymbol{H}}=\left[\overline{\boldsymbol{m}}(\overline{\boldsymbol{H}})+<\frac{\partial \overline{\boldsymbol{H}}}{\partial \dot{\overline{\boldsymbol{Q}}}} \overline{\boldsymbol{U}}_{v}>\right] \mathrm{d} t+\overrightarrow{\boldsymbol{o}}(\overline{\boldsymbol{H}}) \mathrm{d} \overline{\boldsymbol{B}}(t)
$$

where $\overline{\boldsymbol{H}}$ is the modal energy vector of the coupled structures, $\boldsymbol{Q}$ is the modal displacement vector, $\overline{\boldsymbol{U}}_{v}$ is the modal control force vector, $\overline{\boldsymbol{m}}$ and $\bar{\sigma}$ are respectively the drift coefficient vector and diffusion coefficient matrix. For system (15) and index (4b), the dynamical programming equation is obtained as

$$
\min _{\mathbf{U}}\left\{L(\overline{\mathbf{H}}, \mathbf{U})+\left(\overline{\mathbf{m}}+<\frac{\partial \overline{\mathbf{H}}}{\partial \overline{\overline{\mathbf{Q}}}} \overline{\mathbf{U}}_{v}>\right)^{T} \frac{\partial V}{\partial \overline{\mathbf{H}}}+\frac{1}{2} \operatorname{tr}\left(\bar{\sigma} \bar{\sigma}^{T} \frac{\partial^{2} V}{\partial \overline{\mathbf{H}}^{2}}\right)\right\}=\lambda
$$

For the function $L$ quadratic in control, the optimal active control is

$$
\mathrm{U}^{*}=-\frac{1}{2} \mathbf{R}_{p}^{-1}\left(\mathbf{P}_{1}^{T} \bar{\Phi}_{1} \frac{\partial \overline{\mathbf{H}}_{1}}{\partial \dot{\overline{\mathbf{Q}}}_{1}} \frac{\partial V}{\partial \overline{\mathbf{H}}_{1}}+\mathbf{P}_{2}^{T} \bar{\Phi}_{2} \frac{\partial \overline{\mathbf{H}}_{2}}{\partial \dot{\overline{\mathbf{Q}}}_{2}} \frac{\partial V}{\partial \overline{\mathbf{H}}_{2}}\right)
$$

The response statistics of the controlled system can be evaluated by substituting the optimal control into Eq. (14) and using the stochastic averaging method. Numerical results are shown in Figs. 4(a) and 4(b).

\section{OPTIMAL SEMI-ACTIVE CONTROL [12-14]}

The semi-active control depends on a smaller power supply and is more significant. The dynamic characteristics of semi-active control devices need to be taken into account by control. The MR damper is a classical semi-active control device, which can been described by the Bingham model or Bouc-Wen model [18, 19]. The MR-TLCD is another semiactive control device.

\section{Semi-Active Control Law Based on the Bingham Model of MR Dampers}

By applying the stochastic averaging method and stochastic dynamical programming principle to system (1) or (2), the optimal active control (7) can be determined. However, The semi-active MR damper cannot always implement the optimal active control law. According to the Bingham model, the control produced by an MR damper is

$$
u_{i}=-c_{i} \dot{Q}_{i}-F_{i} \operatorname{sgn}\left(\dot{Q}_{i}\right)
$$

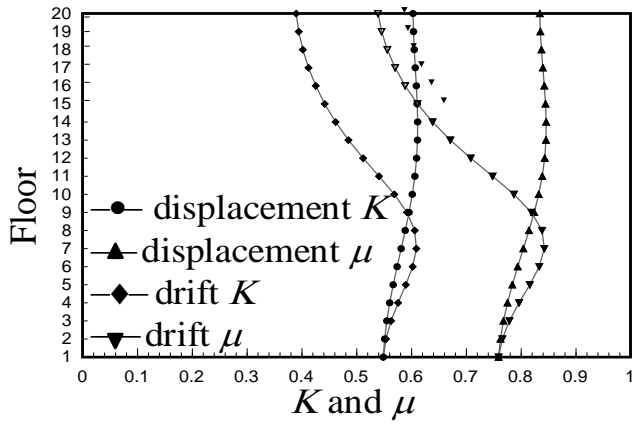

(a) 20-storey structure

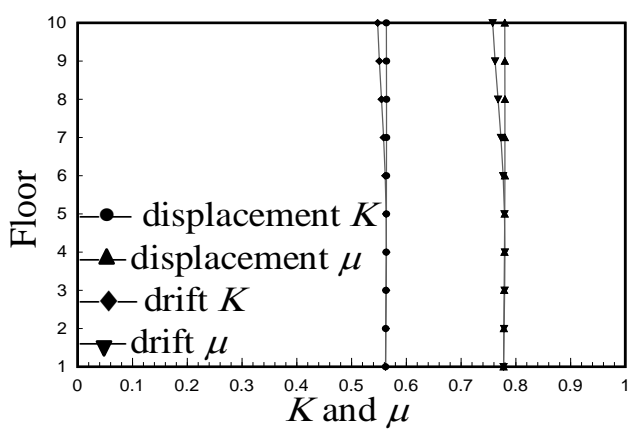

(b) 10-storey structure

Fig. (4). Control effectiveness $(K)$ and efficiency $(\mu)$ of the coupled adjacent structures.

The first part of control (18) is a passive control component which can be incorporated in system, and the second part of control (18) is a semi-active control component which has the amplitude $F_{i}$ adjustable by the applied voltage and is in the opposite direction of velocity. If the semi-active control (18) disagrees with the optimal control, it is set to be zero. Therefore, the optimal semi-active control is

$$
\begin{aligned}
& u_{i}^{s}= \begin{cases}-F_{i}^{*} \operatorname{sgn}\left(\dot{Q}_{i}\right), & F_{i}^{*} \geq 0 \\
0, & F_{i}^{*}<0\end{cases} \\
& F_{i}^{*}=\frac{1}{2} R_{i j}^{-1} \frac{\partial H_{r}}{\partial P_{j}} \frac{\partial V}{\partial H_{r}} \operatorname{sgn}\left(\dot{Q}_{i}\right)
\end{aligned}
$$

The efficacy of semi-active control (19) is generally lower than that of the corresponding active control (7). However, under certain conditions, the relation $F_{i}^{*} \geq 0$ always holds so that the semi-active control (19) agrees with the active control (7). Thus the semi-active MR dampers can perform the optimal active control law.

\section{Semi-Active Control Law Based on the Bouc-Wen Model of MR Dampers}

The Bouc-Wen model can quite describe the dynamic characteristics of MR dampers such as hysteresis. According to this model, the control produced by an MR damper is

$$
\begin{aligned}
u_{i} & =-c_{i} \dot{Q}_{i}-\alpha_{i} z_{i} \\
\dot{z}_{i} & =A_{i} \dot{Q}_{i}-\beta_{i} \dot{Q}_{i}\left|z_{i}\right|^{n_{i}}-\gamma_{i}\left|\dot{Q}_{i}\right| z_{i}\left|z_{i}\right|^{n_{i}-1}
\end{aligned}
$$


The coefficients $c_{i}$ and $\alpha_{i}$ can be separated into two parts, respectively. The first parts $c_{i p}$ and $\alpha_{i p}$ are constants, and the second parts are proportional to the applied voltage $U_{i}$. That is

$$
c_{i}=c_{i p}+c_{i s} U_{i}, \quad \alpha_{i}=\alpha_{i p}+\alpha_{i s} U_{i}
$$

Substituting semi-active control (20) and (21) into system (2), converting it into equivalent non-hysteretic system and incorporating the passive control component in the system yield

$$
\begin{aligned}
& \dot{Q}_{i}=\frac{\partial H}{\partial P_{i}} \\
& \dot{P}_{i}=-\frac{\partial H}{\partial Q_{i}}-\bar{c}_{i j} \frac{\partial H}{\partial P_{j}}+f_{i k} W_{k}(t)-\left(\bar{c}_{i s}+\frac{\partial V_{i s}}{\partial Q_{i}}\right) U_{i}
\end{aligned}
$$

By using the stochastic averaging method and stochastic dynamical programming, the optimal semi-active control voltage is obtained as

$$
U_{i}^{*}=\frac{1}{2 R_{i i}} \bar{c}_{i s}\left(\dot{Q}_{i}\right)^{2} \sum_{r} \frac{\partial V}{\partial H_{r}}
$$

If the right-hand side of Eq. (23) is negative, voltage $U_{i}^{*}$ is set to be zero. Under certain conditions, the right-hand side of Eq. (23) can be always non-negative. Fig. (5) shows the control effectiveness and efficiency of a single-degreeof-freedom system.

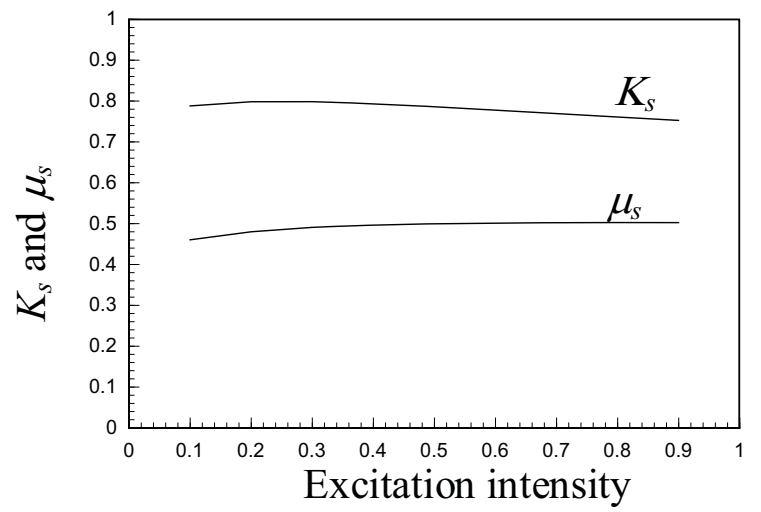

Fig. (5). Semi-active control effectiveness $\left(K_{s}\right)$ and efficiency $\left(\mu_{s}\right)$ based on the Bouc-Wen model.

\section{Semi-Active Control Law of MR-TLCD}

MR-TLCD is a new semi-active control device combining magneto-rheological fluid and tuned liquid column damper, which can be installed at the top floor of buildings for vibration control. A controlled building structure with an MR-TLCD under wind loading can be expressed as

$$
\begin{aligned}
& \boldsymbol{M} \ddot{\boldsymbol{X}}+\boldsymbol{C} \dot{\boldsymbol{X}}+\boldsymbol{K} \boldsymbol{X}=\boldsymbol{F}_{w}(t)+\boldsymbol{E}_{1} f_{D} \\
& m_{D} \ddot{y}+u(\dot{y})+k_{D} y=-\lambda m_{D} \boldsymbol{E}_{1}^{T} \ddot{\boldsymbol{X}}
\end{aligned}
$$

Eqs. (24a) and (24b) are respectively the building and MR-TLCD equations of motion. $f_{D}$ is the interaction force. $F_{w}(t)$ is the wind excitation with the Davenport spectrum. $u$ is the control force produced by the MR fluid, which can be separated into passive part $u_{p}$ and semi-active part $u_{s}$. Making the modal transformation of (24a), combining it with (24b) and linearizing statistically yield

$$
\dot{\boldsymbol{Z}}=\boldsymbol{A} \boldsymbol{Z}+\boldsymbol{F}(t)+\boldsymbol{U}
$$

According to the stochastic dynamical programming principle, the optimal semi-active control obtained is

$$
\begin{aligned}
& u_{s}^{*}= \begin{cases}F^{*} \operatorname{sgn}(\dot{y}), & F^{*} \geq 0 \\
0, & F^{*}<0\end{cases} \\
& F^{*}=\boldsymbol{R}^{-1} \boldsymbol{B}^{T} \boldsymbol{P} \boldsymbol{Z} \operatorname{sgn}(\dot{y})
\end{aligned}
$$

By substituting the semi-active control into Eq. (24) and using the statistical linearization method, the response of the controlled system can be evaluated. Numerical results are shown in Fig. (6).

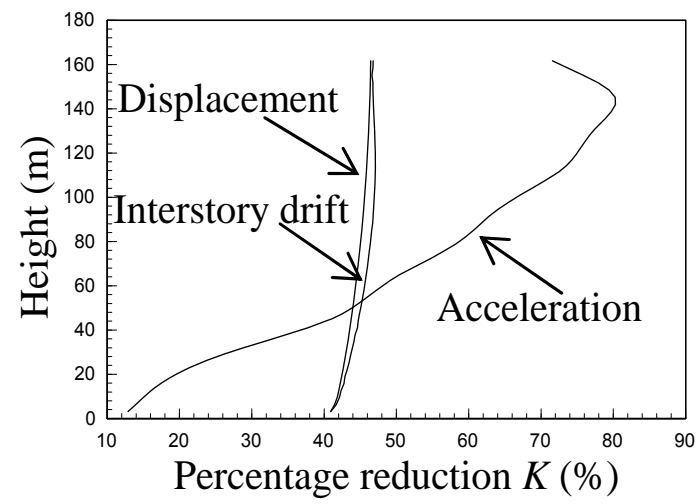

Fig. (6). Control effectiveness of the 51-storey building with MR-TLCD.

\section{DEVELOPMENTS OF STOCHASTIC OPTIMAL CONTROL METHOD [15-17]}

In fact, an optimal feedback control is affected by actuator saturation, partial state observation and control time delay. The control method needs to take these factors into account.

\section{Stochastic Optimal Control Law for Actuator Saturation}

The symmetric bounded control constraint can be expressed as

$\left|u_{i}\right| \leq b_{u i}$

Under this condition, the optimal bounded control derived from (7) is

$$
\begin{aligned}
u_{i}^{*} & = \begin{cases}-F_{i}, & \left|F_{i}\right|<b_{u i} \\
-b_{u i} \operatorname{sgn}\left(F_{i}\right), & \left|F_{i}\right| \geq b_{u i}\end{cases} \\
F_{i} & =\frac{1}{2} R_{i j}^{-1} \frac{\partial H_{r}}{\partial P_{j}} \frac{\partial V}{\partial H_{r}}
\end{aligned}
$$

Numerical results show that control (28) has higher efficiency than the corresponding bang-bang control (Fig. 7) and can attenuate the chattering of bang-bang control (Fig. 8). 


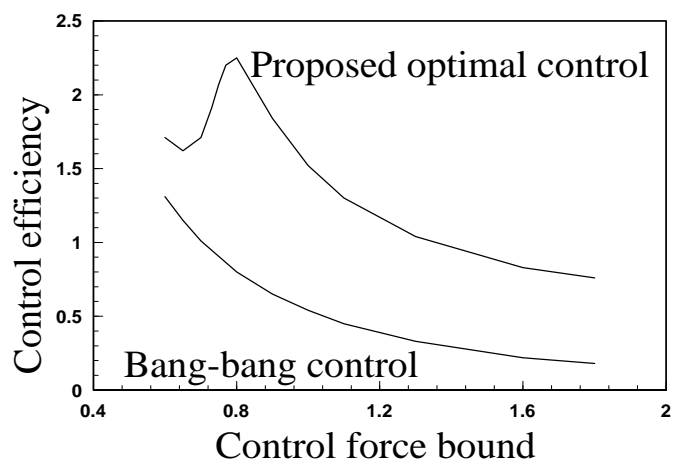

Fig. (7). Control efficiency of proposed stochastic optimal bounded control and bang-bang control.

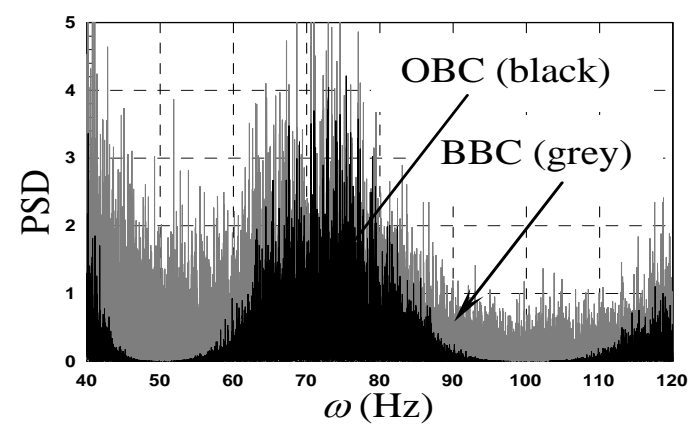

Fig. (8). Power spectral density (PSD) of acceleration responses of bang-bang control (BBC) and proposed stochastic optimal bounded control (OBC).

\section{Stochastic Optimal Control Law for Partially Observable Systems}

Actual control systems are partially observable due to inevitable state measuring and estimating errors. The stochastic optimal control problem of partially observable systems can be converted into that of completely observable systems by the separation theorem. The stochastic optimal control problem of a partially observable system can be expressed as

$$
\begin{aligned}
& \mathrm{d} \boldsymbol{X}=[\boldsymbol{A} \boldsymbol{X}+\boldsymbol{G}(\boldsymbol{X})] \mathrm{d} t+\boldsymbol{B} \boldsymbol{u} \mathrm{d} t+\boldsymbol{C}_{1} \mathrm{~d} \boldsymbol{B}(t) \\
& \mathrm{d} \boldsymbol{Y}=[\boldsymbol{D} \boldsymbol{X}+\boldsymbol{E}(\boldsymbol{X})] \mathrm{d} t+\boldsymbol{F} \boldsymbol{u} \mathrm{d} t+\boldsymbol{C}_{2} \mathrm{~d} \boldsymbol{B}+\boldsymbol{C}_{3} \mathrm{~d} \boldsymbol{B}_{1}(t) \\
& J=E\left\{\int_{0}^{t_{f}} L(\mathbf{X}, \mathbf{u}) \mathrm{d} t+\Psi\left(\mathbf{X}\left(t_{f}\right)\right)\right\}
\end{aligned}
$$

Eqs. (29a), (29b) and (29c) are the system equation of motion, observation equation and control performance index, respectively. If Eqs. (29a) and (29b) are linear, the partially observable control problem (29) can be converted into the following completely observable control problem:

$$
\begin{aligned}
& \mathrm{d} \hat{\boldsymbol{X}}=(\boldsymbol{A} \hat{\boldsymbol{X}}+\boldsymbol{B} \boldsymbol{u}) \mathrm{d} t+\left(\boldsymbol{R}_{C} \boldsymbol{D}^{T}+\boldsymbol{C}_{1} \boldsymbol{C}_{2}^{T}\right) \boldsymbol{C}^{-1} \mathrm{~d} \hat{\boldsymbol{B}}(t) \\
& J_{1}=E\left\{\int_{0}^{t_{f}} L(\hat{\mathbf{X}}, \mathbf{u}) \mathrm{d} t+\Psi_{1}\left(\hat{\mathbf{X}}\left(t_{f}\right)\right)\right\}
\end{aligned}
$$

where $\hat{\boldsymbol{X}}$ is the optimal estimation of $\boldsymbol{X}$. Estimation error $\widetilde{\boldsymbol{X}}=\boldsymbol{X}-\hat{\boldsymbol{X}}$ is the Gaussian process with covariance $\boldsymbol{R}_{C}$ satisfying
$\dot{\boldsymbol{R}}_{C}=A \boldsymbol{R}_{C}+\boldsymbol{R}_{C} A^{T}-\left(\boldsymbol{R}_{C} D^{T}+C_{1} C_{2}^{T}\right) C^{-1}\left(D R_{C}+C_{2} C_{1}^{T}\right)+C_{1} C_{1}^{T}$

The optimal control of completely observable system (30) can be determined by using the method above.

If Eqs. (29a) and (29b) are nonlinear, separate control into two parts, i.e., $\boldsymbol{u}=\boldsymbol{u}_{1}+\boldsymbol{u}_{2}$ and select $\boldsymbol{u}_{1}$ such that $\boldsymbol{G}+\boldsymbol{B} \boldsymbol{u}_{1}$ and $\boldsymbol{E}+\boldsymbol{F} \boldsymbol{u}_{1}$ are potential functions, and then convert the partially observable control problem (29) into the following completely observable control problem:

$$
\begin{aligned}
& \mathrm{d} \hat{\boldsymbol{X}}=\left(\boldsymbol{A} \hat{\boldsymbol{X}}+\boldsymbol{B} \boldsymbol{u}_{2}\right) \mathrm{d} t+\left(\boldsymbol{R}_{C} \boldsymbol{D}^{T}+\boldsymbol{C}_{1} \boldsymbol{C}_{2}^{T}\right) \boldsymbol{C}^{-1} \mathrm{~d} \boldsymbol{V}_{I} \\
& \mathrm{~d} \boldsymbol{V}_{I}=\mathrm{d} \boldsymbol{Y}-\boldsymbol{D} \hat{\boldsymbol{X}} \mathrm{d} t \\
& J_{2}=E\left\{\int_{0}^{T} L_{2}\left(\hat{\mathbf{X}}, \mathbf{u}_{2}\right) \mathrm{d} t+\Psi_{2}\left(\hat{\mathbf{X}}\left(t_{f}\right)\right)\right\}
\end{aligned}
$$

where $V_{I}$ is the innovation process. The optimal control of completely observable system (32) can be determined similarly. Numerical results are shown in Fig. (9).

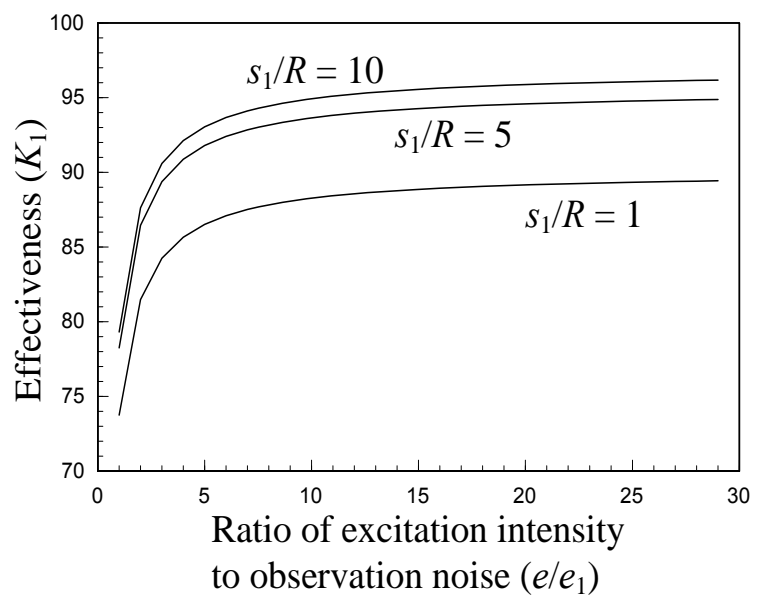

Fig. (9). Control effectiveness of the partially observable nonlinear system.

\section{Stochastic Optimal Time-Delayed Control Law}

The optimal time-delay control problem can be expressed as

$\dot{Q}_{i}=\frac{\partial H}{\partial P_{i}}$

$\dot{P}_{i}=-\frac{\partial H}{\partial Q_{i}}-c_{i j} \frac{\partial H}{\partial P_{j}}+f_{i k} \xi_{k}(t)+u_{i}\left(Q_{\tau i}, P_{\tau i}\right)$

$J=\lim _{t_{f} \rightarrow \infty} \frac{1}{t_{f}} \int_{0}^{t_{f}} L\left(Q_{i}, P_{i}, u_{i}\right) \mathrm{d} t$

where $\tau$ is the time delay in control. Based on the stochastic averaging method for time-delayed systems, the state transformation is obtained as

$Q_{\tau i}=Q_{i}(t-\tau) \doteq Q_{i}(t) \cos \omega_{i} \tau-\frac{P_{i}(t)}{\omega_{i}} \sin \omega_{i} \tau$ 


$$
P_{\tau i}=P_{i}(t-\tau) \doteq Q_{i}(t) \omega_{i} \sin \omega_{i} \tau+P_{i}(t) \cos \omega_{i} \tau
$$

and the Itô stochastic differential equation is

$$
\mathrm{d} H_{r}=\left[m_{r}(\mathbf{H})+<u_{i} \frac{\partial H_{r}}{\partial P_{i}}>\right] \mathrm{d} t+\sigma_{r k}(\mathbf{H}) \mathrm{d} B_{k}(t)
$$

The corresponding performance index for infinite timeinterval ergodic control is

$$
J=\lim _{t_{f} \rightarrow \infty} \frac{1}{t_{f}} \int_{0}^{t_{f}} L\left(H_{r}, u_{i}\right) \mathrm{d} t
$$

Eq. (35) is a non-time-delay optimal control problem and its optimal control can be determined by using the method above. The inverse transformation of (34) is

$$
\begin{aligned}
& Q_{i}=Q_{i}(t) \doteq Q_{\tau i} \cos \omega_{i} \tau+\frac{P_{\tau i}}{\omega_{i}} \sin \omega_{i} \tau \\
& P_{i}=P_{i}(t) \doteq-Q_{\pi i} \omega_{i} \sin \omega_{i} \tau+P_{\tau i} \cos \omega_{i} \tau
\end{aligned}
$$

Using (36) yields the optimal time-delayed control

$$
u_{i}^{*}=-\frac{1}{2} R_{i j}^{-1}\left(\frac{\partial H_{r}}{\partial P_{j}} \frac{\partial V}{\partial H_{r}}\right)_{\tau}
$$

where $(\cdot)_{\tau}$ represents the time-delayed state function. These control methods have respectively taken into account the effects of actuator saturation, partial state observation and control time delay, and however, need to improve further.

\section{ACKNOWLEDGEMENT}

This study was supported by the Zhejiang Provincial Natural Science Foundation of China under grant no. Y607087.

\section{REFERENCES}

[1] G.W. Housner, L.A. Bergman, T.K. Caughey, A.G. Chassiakos, R.O. Claus, S.F. Masri, R.E. Skelton, T.T. Soong, B.F. Spencer and J.T.P Yao, "Structural control: past, present, and future", ASCE International Journal of Engineering Science, 123(9): 897-971, 1997.
[2] W.H. Fleming and R.W. Rishel, Deterministic and Stochastic Optimal Control, Berlin: Springer-Verlag, 1975.

[3] R. F. Stengel, Stochastic Optimal Control: theory and application, New York: John Wiley \& Sons, 1986.

[4] M.F. Dimentberg, D.V. Iourtchenko and A.S. Bratus, "Optimal bounded control of steady-state random vibrations", Probabilistic Engineering Mechanics, 15: 381-6, 2000.

[5] T.T. Soong, Active Structural Control: theory and practice, New York: John Wiley \& Sons, 1990.

[6] J.N. Yang, A.K. Agrawal and S. Chen,"'Optimal polynomial control for seismically excited non-linear and hysteretic structures", Earthquake Engineering and Structural Dynamics, 25: 1211-30, 1996.

[7] L.G. Crespo and J.Q. Sun, "Stochastic Optimal Control of Nonlinear Systems via Short-time Gaussian approximation and Cell Mapping", Nonlinear Dynamics, 28: 323-42, 2002.

[8] W.Q. Zhu and Z.G. Ying, "Optimal Nonlinear Feedback Control of Quasi-Hamiltonian Systems", Science in China, Series A, 42: 1213-9, 1999.

[9] W.Q. Zhu, Z.G. Ying and T.T. Soong, "An Optimal Nonlinear Feedback Control Strategy for Randomly Excited Structural Systems", Nonlinear Dynamics, 24: 31-51, 2001.

[10] W.Q. Zhu, Z.G. Ying, Y.Q. Ni and J.M. Ko, "Optimal nonlinear stochastic control of hysteretic systems", ASCE International Journal of Engineering Science, 126: 1027-32, 2000.

[11] Z.G. Ying, Y.Q. Ni and J.M. Ko, "Non-linear Stochastic Optimal Control for Coupled-structures System of Multi-degree-offreedom", Journal of Sound and Vibration, 274: 843-61, 2004.

[12] Z.G. Ying, W.Q. Zhu and T.T. Soong, "A Stochastic Optimal semi-active Control Strategy for ER/MR Dampers", Journal of Sound and Vibration, 259: 45-62, 2003.

[13] Z.G. Ying, Y.Q. Ni and J.M. Ko, “A New Stochastic Optimal Control Strategy for Hysteretic MR Dampers", Acta Mechanical Solida Sinica, 17: 223-9, 2004.

[14] Z.G. Ying, Y.Q. Ni and J.M. Ko, "Semi-active Optimal Control of Linearized Systems with Multi-degree of Freedom and Application', Journal of Sound and Vibration, 279: 373-88, 2005.

[15] Z.G. Ying and W.Q. Zhu, “A Stochastically Averaged Optimal Control Strategy for Quasi Hamiltonian Systems with Actuator Saturation', Automatica, 42: 1577-82, 2006.

[16] W.Q. Zhu and Z.G. Ying, "Nonlinear Stochastic Optimal Control of Partially Observable Linear Structures", Structural Engineering, 24: 333-42, 2002.

[17] Z.G. Ying and W.Q. Zhu, "A Stochastic Optimal Control Strategy for Partially Observable Nonlinear Quasi-Hamiltonian Systems", Journal of Sound and Vibration, 310: 184-96, 2008.

[18] S.J. Dyke, B.F. Spencer, M.K. Sain and J.D.Carlson," Modeling and Control of Magnetorheological Dampers for Seismic Response Reduction", Smart Materials and Structures, 5: 565-75, 1996.

[19] M.D. Symans and M.C. Constantinon, "Semi-active Control Systems for Seismic Protection of Structures: a State-of-the-art Review", Structural Engineering, 21: 469-87, 1999.

\section{(C) Z.G. Ying; Licensee Bentham Open.}

This is an open access article licensed under the terms of the Creative Commons Attribution Non-Commercial License (http://creativecommons.org/licenses/by-nc/3.0/) which permits unrestricted, non-commercial use, distribution and reproduction in any medium, provided the work is properly cited. 